\title{
Intermittent permeation of cylindrical nanopores by water
}

\author{
Rosalind Allen, ${ }^{1,}$ f Simone Melchionna, ${ }^{2}$ and Jean-Pierre Hansen ${ }^{1}$ \\ ${ }^{1}$ Department of Chemistry, University of Cambridge, \\ Lensfield Road, Cambridge CB2 1EW, United Kingdom \\ ${ }^{2}$ Istituto per le applicazioni del calculo "M. Picone", \\ Consiglio Nazionale delle Ricerche, V. le del Policlinico 137, 00161 Rome, Italy
}

(Dated: October 27, 2018)

\begin{abstract}
Molecular Dynamics simulations of water molecules in nanometre sized cylindrical channels connecting two reservoirs show that the permeation of water is very sensitive to the channel radius and to electric polarization of the embedding material. At threshold, the permeation is intermittent on a nanosecond timescale, and strongly enhanced by the presence of an ion inside the channel, providing a possible mechanism for gating. Confined water remains surprisingly fluid and bulk-like. Its behaviour differs strikingly from that of a reference Lennard-Jones fluid, which tends to contract into a highly layered structure inside the channel.
\end{abstract}

Highly confined molecular or ionic fluids, including fluid films compressed by surface force machines [1], or trapped in nanoporous materials like zeolites [2] or carbon nanotubes [3, 4], exhibit distinctive structural, dynamical and phase behaviour which may differ widely from their corresponding bulk properties. Recent experiments [5] and simulations [4] demonstrate the interesting behaviour of water under conditions of extreme confinement, which is related to the disruption of the hydrogenbond network between $\mathrm{H}_{2} \mathrm{O}$ molecules, depending on the hydrophobic or hydrophilic nature of the confining surface. A good understanding of the molecular organization of confined water is essential to the study of the selectivity and permeation mechanisms of ion channels through membranes [6].

While much of the published simulation work is on realistic or semi-realistic models of specific ion channels [7], the present letter examines the generic behaviour of water permeation in a highly simplified model of a channel, as a function of a small number of physical parameters characterizing the channel. The proper inclusion of end effects and Coulombic interactions increases the relevance of the present results for real ion channels, compared to earlier studies of infinite channels [8, 9, 10].

The model considered in the present work is that of a cylindrical channel of radius $R$ and length $L$ through a slab of dielectric material, of relative permittivity $\epsilon$ (representing the membrane), which separates two reservoirs containing water molecules and eventually ions (representing the intra and extra-cellular compartments), symmetrically placed on both sides of the slab(see Fig. 11). In our Molecular Dynamics (MD) simulations 11, the two reservoirs, which are connected through a common $\mathrm{x}-\mathrm{y}$ plane under periodic boundary conditions, contain typically $N=500$ water molecules each. The number of water molecules in the reservoirs fluctuates somewhat

*Electronic address: rja22@cam.ac.uk

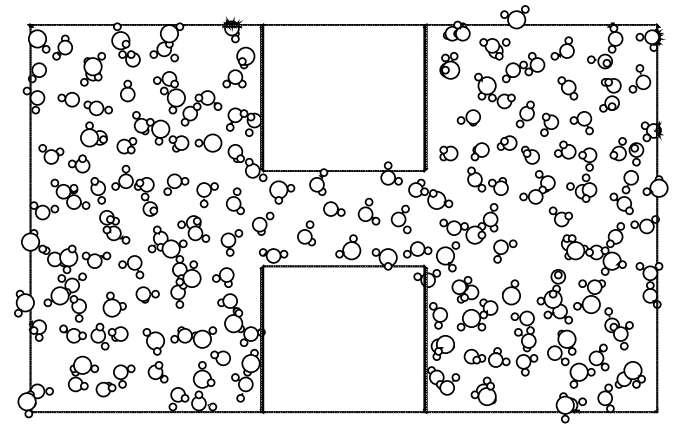

FIG. 1: Schematic representation of a cut through the simulation cell. The $z$-axis coincides with the axis of the cylindrical channel connecting the two reservoirs; the interior wall region is a continuum of permittivity $\epsilon$. Periodic boundary conditions apply in all three directions. The channel radius and length are shown at their effective values, $R_{\text {eff }}<R$ and $L_{e f f}>L$, as explained in the text.

when the cylindrical channel fills or empties; in order to maintain a constant density of $0.996 \mathrm{gr} \mathrm{cm}^{-3}$, the reservoir length parallel to the axis of the channel (taken to be $z$ ) and the z-components of the molecular position vectors are scaled regularly using a version of the Berendsen barostat 12].

The water molecules interact via the standard SPC/E pair potential [13] which involves a Lennard-Jones (LJ) potential between the O-atoms $(\sigma=0.3169 \mathrm{~nm}, \epsilon=$ $0.6502 \mathrm{~kJ} \mathrm{~mol}^{-1}$ i.e $\epsilon / k_{B}=78.2 \mathrm{~K}$ ), and Coulombic interactions between the sites associated with the $\mathrm{O}$ atoms $(q=-0.8476)$ and the two $\mathrm{H}$ atoms $(q=+0.4238)$. The simulations reported in this letter contain no ions or just one $\mathrm{K}^{+} \mathrm{Cl}^{-}$pair, which interact with the water molecules as in ref. [8]. The radial interaction between the channel surface and the water molecules is taken to be the LJ potential between a $\mathrm{CH}_{3}$ group and an $\mathrm{O}$ atom [14], integrated over the surface of an infinitely long cylinder $1 \mathbf{1 5}$. 
The same potential, now as a function of the distance from the planar wall, is used for the membrane-water interaction, with an appropriate rounding and shifting procedure to avoid any discontinuities. A similar procedure is used for ion-surface interactions.

Since water molecules and ions carry charged sites, they polarize the membrane modelled here by the dielectric slab. The interface between slab, channel or reservoir is treated as infinitely sharp (dielectric discontinuity), so that the polarization charge is entirely localized on the surface of the slab and can be calculated by the variational procedure described in ref. [16]; the numerical implementation requires the use of a grid spanning the cylindrical surface and the planar interface between the slab and the reservoirs. Most of the results presented in this letter neglect membrane polarization, i.e. $\epsilon=1$ is assumed; the importance of polarization on the behaviour of confined water is assessed later in the letter, by comparing data obtained for $\epsilon=1$ and $\epsilon=4$.

The present work is restricted to equilibrium fluctuations. Starting from initial water configurations with an empty channel, the number of molecules within the channel is monitored as a function of time, for total times up to 6.5 ns. Simulations were carried out for a rather short channel of length $L=0.8 \mathrm{~nm}$. The channel radius $R$ was varied between 0.4 and $0.7 \mathrm{~nm}$, but in view of the soft nature of the surface-water interaction, the corresponding effective radii of the cylinders accessible to the centres of the $\mathrm{O}$ atoms (i.e. such that the wall-O repulsive potential is less than $k_{B} T$ ) are $R_{e f f}=0.17-0.47 \mathrm{~nm}$. Similarly the effective length of the channel is $L_{e f f}=1.26 \mathrm{~nm}$, which is roughly the length of the selectivity filter in the KcsA channel, the structure of which has recently been resolved by X-ray diffraction [17, 18].

When the channels are filled, the radial density profiles of $\mathrm{O}$ and $\mathrm{H}$ atoms can be calculated by averaging over all water configurations generated in the MD runs. In order to gain a better understanding of the importance of the hydrogen-bond network within the channel, simulations were also carried out for a reference system of non-associative molecules obtained by removing the $\mathrm{H}$ atoms, i.e. an imaginary system of "OW" atoms interacting solely through the Lennard-Jones part of the SPC/E potential. While the $\mathrm{H}_{2} \mathrm{O}$ simulations were run at room temperature, the reference runs for the $\mathrm{OW}$ atoms were carried out at $T=120 \mathrm{~K}$ and a reduced density $\rho^{\star}=n \sigma^{3} / V=0.85$, conditions under which the bulk LJ system is expected to be liquid. Under these conditions we also find liquid-like diffusion in the pore, whereas at the lower temperatures $T=56.2 \mathrm{~K}$ (i.e. $T^{*}=k_{B} T / \epsilon=0.72$, close to the LJ triple point 19]) and $T=85 \mathrm{~K}$ the $\mathrm{OW}$ atoms form a disordered solid in the pore. The number $n(t)$ of $\mathrm{OW}$ atoms within channels of two different radii are shown in Fig. 2 as a function of time. As expected, there are large fluctuations in $n(t)$, but the key observation is that the LJ particles fill the

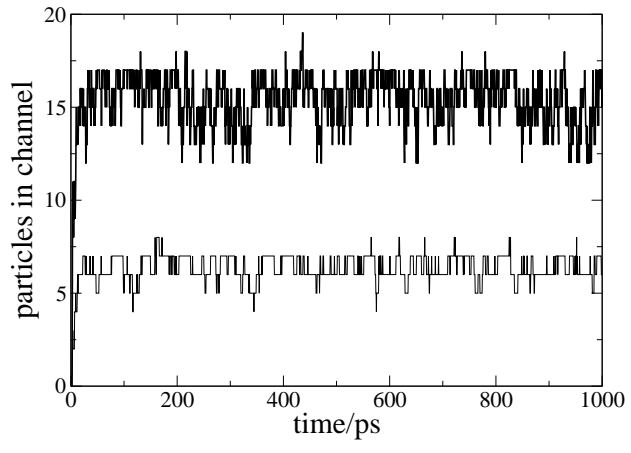

FIG. 2: Number $n(t)$ of OW atoms inside channels of radius $R=0.45 \mathrm{~nm}\left(R_{e f f}=0.22 \mathrm{~nm}\right)$ and $R=0.6 \mathrm{~nm}\left(R_{e f f}=\right.$ $0.37 \mathrm{~nm}$ ), in bold, as a function of time (in ps).

channel for all radii considered. The mean density of particles inside the channel, $\langle n(t)\rangle /\left(\pi R_{\text {eff }}^{2} L\right)$ is about 1.7 times larger than the bulk density in the reservoirs, but the confined atoms remain in a fluid state, as characterized by a diffusion constant along the cylinder axis $\left(2 \times 10^{-5} \mathrm{~cm}^{2} \mathrm{~s}^{-1}\right)$ comparable to the bulk diffusion constant $\left(6 \times 10^{-5} \mathrm{~cm}^{2} \mathrm{~s}^{-1}\right)$. The radial density profile $\rho(r)$ is highly structured, as shown in Fig. 4d, indicative of layering.

The behaviour changes dramatically when the OW atoms are replaced by $\mathrm{H}_{2} \mathrm{O}$ molecules. The numbers of molecules inside the channel are shown as a function of time in Fig. 3 for four different cylinder radii. When $R=0.55 \mathrm{~nm}$, the channel shows no sign of filling and the small fluctuations in $n(t)$ away from zero reflect attempts by small numbers of molecules to penetrate the channel at either end. At the slightly larger channel radius $R=0.6 \mathrm{~nm}$, a strikingly intermittent behaviour is apparent, reminiscent of recent observations in simulations of water in carbon nanotubes [4] and of water in atomistically rough models of ion channels [6]. States where the channel is filled alternate stochastically with empty channel states, on a time scale of typically $1 \mathrm{~ns}$. When the radius is further increased to $R=0.65 \mathrm{~nm}$, the channel appears to remain filled after an initial intermittency, although it cannot be ruled out that short periods in the empty state could be observed in much longer runs. Finally at $R=0.7 \mathrm{~nm}$, the channel appears to remain filled throughout, with a mean density of $0.034 \AA^{-3}$ comparable to the bulk density in the reservoirs, $0.033 \AA^{-3}$. Diffusion of the confined molecules along the $z$-axis occurs on a timescale comparable to bulk diffusion (the reservoir diffusion constant and the channel $z$-axis diffusion constant both being $3 \times 10^{-5} \mathrm{~cm}^{2} \mathrm{~s}^{-1}$ ). Radial density profiles of the $\mathrm{O}$ and $\mathrm{H}$ atoms, averaged over occupied states of the channel, are shown in Fig.4 a-c for three values of the radius $R$. They are surprisingly flat, and contrast sharply with the density profile of the LennardJones particles plotted in Fig. 4d. The absence of a clear-cut layering of the $\mathrm{H}_{2} \mathrm{O}$ molecules, as well as the 

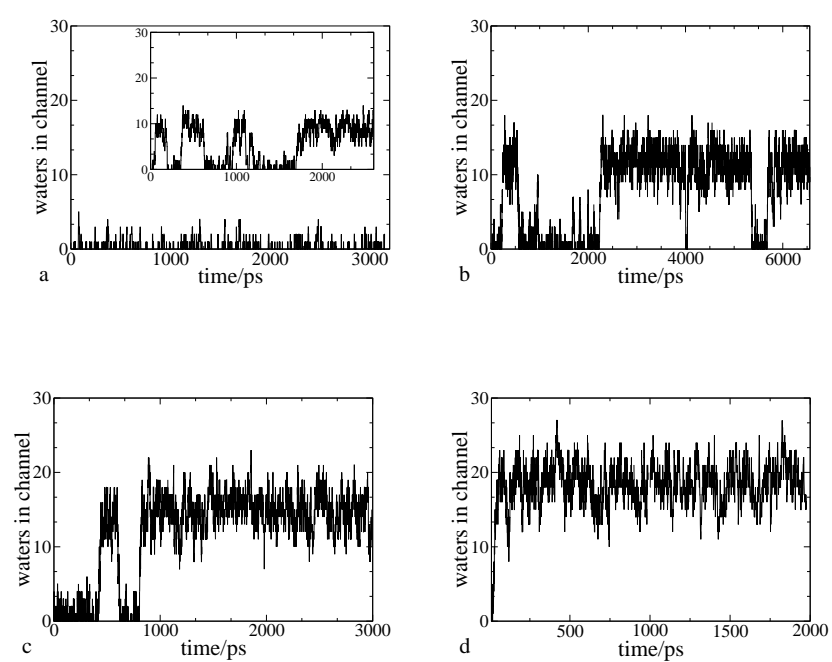

FIG. 3: Number $n(t)$ of water molecules inside channels of length $L=0.8 \mathrm{~nm}\left(L_{\text {eff }}=1.26 \mathrm{~nm}\right)$ and radii $R=0.55 \mathrm{~nm}$ $\left(R_{e f f}=0.32 \mathrm{~nm}\right)(\mathrm{a}), R=0.6 \mathrm{~nm}\left(R_{e f f}=0.37 \mathrm{~nm}\right)(\mathrm{b})$, $R=0.65 \mathrm{~nm}\left(R_{e f f}=0.42 \mathrm{~nm}\right)(\mathrm{c})$ and $R=0.7 \mathrm{~nm}\left(R_{e f f}=\right.$ $0.47 \mathrm{~nm}$ ) (d) as a funtion of time (in ps); note that the total durations of the four runs vary; all four runs are for $\epsilon=1$; the inset in frame (a) shows $n(t)$ for the same $L$ and $R$, but with $\epsilon=4$.

substantial fluidity of the confined water, characterized by the bulk-like diffusion, agree qualitatively with recent surface force apparatus measurements of highly confined water films河.

The results in Fig. 3 show that the filling (or wetting) of a cylindrical channel is very sensitive to its radius, with a threshold radius below which water does not penetrate on this timescale. So far the polarization of the confining protein and membrane has been neglected, by setting $\epsilon=1$. One intuitively expects that such polarization by the charge distribution on the water molecules will lower the electrostatic energy, and hence favour the wetting of narrow channels. This is confirmed by the time trace of $n(t)$ shown in the inset to Fig. 3a from MD simulations with $\epsilon=4$ : water molecules are seen to fill the $R=0.55 \mathrm{~nm}$ channel intermittently, while the same channel remains empty in the absence of membrane polarization. The latter effect is thus found to lower the threshold radius above which water molecules can penetrate inside the channel. The effect is expected to be significantly enhanced for larger values of $\epsilon$, which may be more appropriate for the channel protein and membrane.

Returning to the non-polarizable case $\epsilon=1$, water can be made to fill a narrow channel by placing an ion inside. In an MD simulation a $K^{+}$ion was held fixed on the axis in the middle of a channel of radius $R=0.5 \mathrm{~nm}$; the channel was then found to be rapidly filled by $\mathrm{H}_{2} \mathrm{O}$ molecules with an average occupation number $n \approx 6.5$. Water molecules were found to penetrate channels even with $R$ as small as $0.3 \mathrm{~nm}$ under these circumstances.
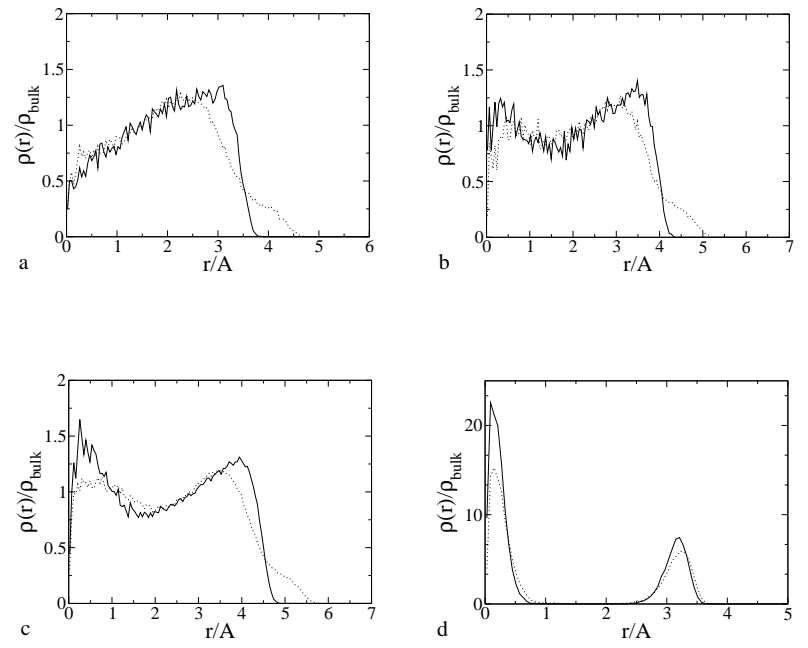

FIG. 4: Radial density profiles $\rho_{\alpha}(r)$ of $\mathrm{O}$ atoms (full curves) and $\mathrm{H}$ atoms (dotted curves) of water in channels of length $L=0.8 \mathrm{~nm}\left(L_{e f f}=1.26 \mathrm{~nm}\right)$ and radii $R=0.6 \mathrm{~nm}\left(R_{e f f}=\right.$ $0.37 \mathrm{~nm})(\mathrm{a}), R=0.65 \mathrm{~nm}\left(R_{e f f}=0.42 \mathrm{~nm}\right)(\mathrm{b})$ and $R=$ $0.7 \mathrm{~nm}\left(R_{e f f}=0.47 \mathrm{~nm}\right)(\mathrm{c})$. Averages are taken only over times when the channel is filled. Frame (d) shows the radial profiles for OW atoms in a channel of the same length and $R=0.6 \mathrm{~nm}\left(R_{e f f}=0.37 \mathrm{~nm}\right)$, at temperatures $T=85 \mathrm{~K}$ (dotted curve) and $T=120 \mathrm{~K}$ (full curve).

A close examination of molecular configurations, radial number density profiles and mean occupation numbers leads to the conclusion that water fills ion channels beyond a critical, $\epsilon$-dependent radius in a surprisingly uniform manner which is intermittent near the threshold. As soon as water wets the channel, the water molecules appear to fill the available volume, with a mean density comparable to the bulk density of liquid water. In this series of simulations, no long-lived single-file chains of water molecules were observed. Using an energetic criterion[20 the mean number of hydrogen bonds was found to be 2.5 in a channel of $R=0.7 \mathrm{~nm}$, compared to 3.5 in the reservoir.

In summary we have investigated a highly simplified, generic model for the selectivity filter of ion channels, which is entirely characterized by three physical parameters $R, L$ and $\epsilon$, once one has fixed the interaction parameters of the wall. The present investigation was limited to $L=0.8 \mathrm{~nm}$. At the threshold radius, water fills the channel intermittently, and the fraction of time over which the channel is in the occupied state depends sensitively on $R$ and $\epsilon$. The timescale for the intermittency is expected to increase with channel length $L$, because larger fluctuations at the channel ends will be required for channel filling. Finite, physiologically relevant concentrations of salt will be included in future work. In view of the preliminary results with one ion fixed within the channel, it is expected that permeation of narrow channels by water molecules and ions is a very cooperative effect, where the polarization of the channel surface will play a crucial 
role, which may provide a simple scenario for ion channel gating.

The authors acknowledge constructive discussions with Ruth Lynden-Bell and Bob Eisenberg. RA is grateful to the EPSRC and to Unilever for a post-graduate CASE award and SM is grateful to the Leverhulme Trust for post-doctoral support during the early stages of this project.

[1] See e.g. J. Israelachvili, Intermolecular and Surface Forces, (Academic Press, New York, 1992).

[2] B. Smit, J. Phys. Chem. 99, 5597 (1995).

[3] M. Wilson and P. A. Madden, J. Am. Chem. Soc. 125, 2101 (2001).

[4] G. Hummer, J. C. Rasaiah and J. P. Nowortya, Nature 414, 188 (2001).

[5] U. Raviv, P. Laurat and J. Klein, Nature 413, 51 (2001).

[6] O. Beckstein, P. C. Biggin and M. S. P. Sansom, J. Phys. Chem. B 105, 12902 (2001).

[7] For a recent review, see J. W. Allen, S. Kury, D. P. Tieleman, P. C. Biggin, G. R. Smith and M. S. P. Sansom, Quart. Rev. Biophys. 34, 473 (2001).

[8] R. Lynden-Bell and J. Rasaiah, J. Chem. Phys. 105, 9266 (1996).

[9] D. Goulding, S. Melchionna and J.-P. Hansen, Phys.
Chem. Chem. Phys. 3, 1644 (2001).

[10] T. W. Allen, S. Kuyucak and S.-H. Chung, J. Chem. Phys. 111, 7985 (1999).

[11] All simulations were performed using the package DL_PROTEIN, written by S. Melchionna and S. Cozzini: S. Melchionna and S. Cozzini, DL_Protein 2.1 User Guide, 2001.

[12] H. J. C. Berendsen, J. P. M. Postma, W. F. van Gunsteren, A. DiNola and J. R. Haak, J. Chem. Phys. 81, 3684 (1984).

[13] H. J. C. Berendsen, J. R. Grigera and T. P. Straatsma, J. Phys. Chem. 91, 6269 (1987).

[14] M G. Martin and J. I. Siepmann, J. Phys. Chem. B 102, 2569 (1998).

[15] B. K. Peterson, J. P. R. B. Walton and K. E. Gubbins, J. Chem. Soc. Faraday Trans. 2 82, 1789 (1986).

[16] R. Allen, J.-P. Hansen and S. Melchionna, Phys. Chem. Chem. Phys. 3, 4177 (2001).

[17] D. A. Doyle, J. M. Cabral, R. A. Pfuetzner, A. L. Kuo, J. M. Gulbis, S. L. Cohen, B. T. Chait and R. MacKinnon, Science 280, 69 (1998).

[18] Y. Zhou, J. H. Morais-Cabral, A. Kaufman and R. MacKinnon, Nature 414, 43 (2001).

[19] J.-P. Hansen and L. Verlet, Phys. Rev. 184, 151 (1969).

[20] W. L. Jorgensen, J. Chandrasekar, J. D. Madura, R. W. Inpey and M. L. Klein, J. Chem. Phys. 79, 926 (1983). 